Musées, Patrimoine et Culture scientifiques et techniques

$127 \mid 2010$

janvier - février 2010

\title{
Art, science et culture scientifique
}

Jean-Yves Bernaud, Michel Brière et Jacques Lochard

URL : http://journals.openedition.org/ocim/182

DOI : $10.4000 /$ ocim. 182

ISSN : 2108-646X

Éditeur

OCIM

Édition imprimée

Date de publication : 1 janvier 2010

Pagination : 5-13

ISSN : 0994-1908

Référence électronique

Jean-Yves Bernaud, Michel Brière et Jacques Lochard, "Art, science et culture scientifique », La Lettre de l'OCIM [En ligne], 127 | 2010, mis en ligne le 01 janvier 2012, consulté le 19 avril 2019. URL : http:// journals.openedition.org/ocim/182 ; DOI : 10.4000/ocim.182 
radioprotection qui s'est tenu en octobre 2008 dans la capitale argentine. De retour en France, après un détour par le Petit Palais à Paris à l'occasion des vœux 2009 de l'IRSN, les œuvres et les bornes ont ensuite été présentées en février 2009 à l'Espace Zoé sur le site du Commissariat à l'Énergie Atomique (CEA) de Fontenay-aux-Roses, lieu hautement symbolique de l'aventure du développement de l'énergie nucléaire : c'est en effet sur ce site que Frédéric Joliot-Curie avec son équipe de pionniers a réussi à faire diverger Zoé, la première pile atomique française, en décembre 1948. Cette étape a donné aux experts de l'IRSN et du CEA l'occasion de s'interroger sur les conditions et les moyens d'une diffusion de la culture de radioprotection auprès du grand public.

Depuis novembre 2009 et jusqu'en avril 2010 l'exposition est présentée à la Fondation Verdan, musée de la Main à Lausanne, puis elle itinèrera au musée Technique d'Helsinki de mai à septembre 2010 et au CCSTI de Chambéry en 2011.

Rayons X, radioactivité, radioprotection, sujets complexes, parfois graves, encore souvent tabous et matières à controverses, sont abordés par l'exposition de manière originale. D'une part en plaçant l'humain, l'intime, au cœur du propos, en inscrivant le récit scientifique dans le contexte de l'histoire politique, sociale et culturelle du XXe siècle et en faisant revivre les personnages, célèbres ou anonymes, qui ont marqué cette aventure. D'autre part, en offrant sur ce récit deux approches croisées, mariant le regard rationnel du scientifique et la vision libre et décalée de l'artiste.

Des bornes vidéos thématiques et des œuvres d'artistes plasticiens évoquent les grandes figures, les grands moments de cette histoire à la fois fantastique et dramatique ainsi que l'atmosphère ambiante : les découvertes des rayons $X$, de la radioactivité et de la fission nucléaire, les développements de l'industrie du radium et de l'industrie nucléaire, les progrès de la radiologie médicale, de la médecine nucléaire et de la radiothérapie, les bombardements d'Hiroshima et de Nagasaki, les essais nucléaires de la Guerre froide et la catastrophe de Tchernobyl. Bornes et œuvres illustrent les grandes étapes du développement de la radioprotection et les moyens techniques mis au point pour mieux se protéger contre les rayonnements artificiels mais aussi les rayons cosmiques et le radon dans les habitations. Entre les bornes et les œuvres, un dialogue s'établit. Comme une histoire racontée à plusieurs voix. Le visiteur est invité à s'immiscer dans l'histoire, à s'approprier l'espace, à y dessiner son propre parcours, au gré de ses questionnements, au diapason de ses émotions.

La réalisation de l'exposition a été le fruit d'une coopération étroite entre l'équipe de production dont faisaient partie évidemment les artistes, le comité scientifique et aussi les équipes de l'IRSN qui, tout au long des trois années du processus de préparation, ont alimenté la réflexion sur les contenus des vidéos. La division « Ouverture à la Société » de l'IRSN en particulier a joué le rôle de médiateur entre l'équipe de production et les experts de l'Institut. L'ensemble des documents scientifiques ont ainsi été validés par des responsables opérationnels.

Les œuvres ont été réalisées par Peter Keene et Piet'sO. Sur le volet artistique il faut également inclure Jean-Yves Pipaud, qui a réalisé les vidéos associées à chacune des œuvres. Chaque pièce de

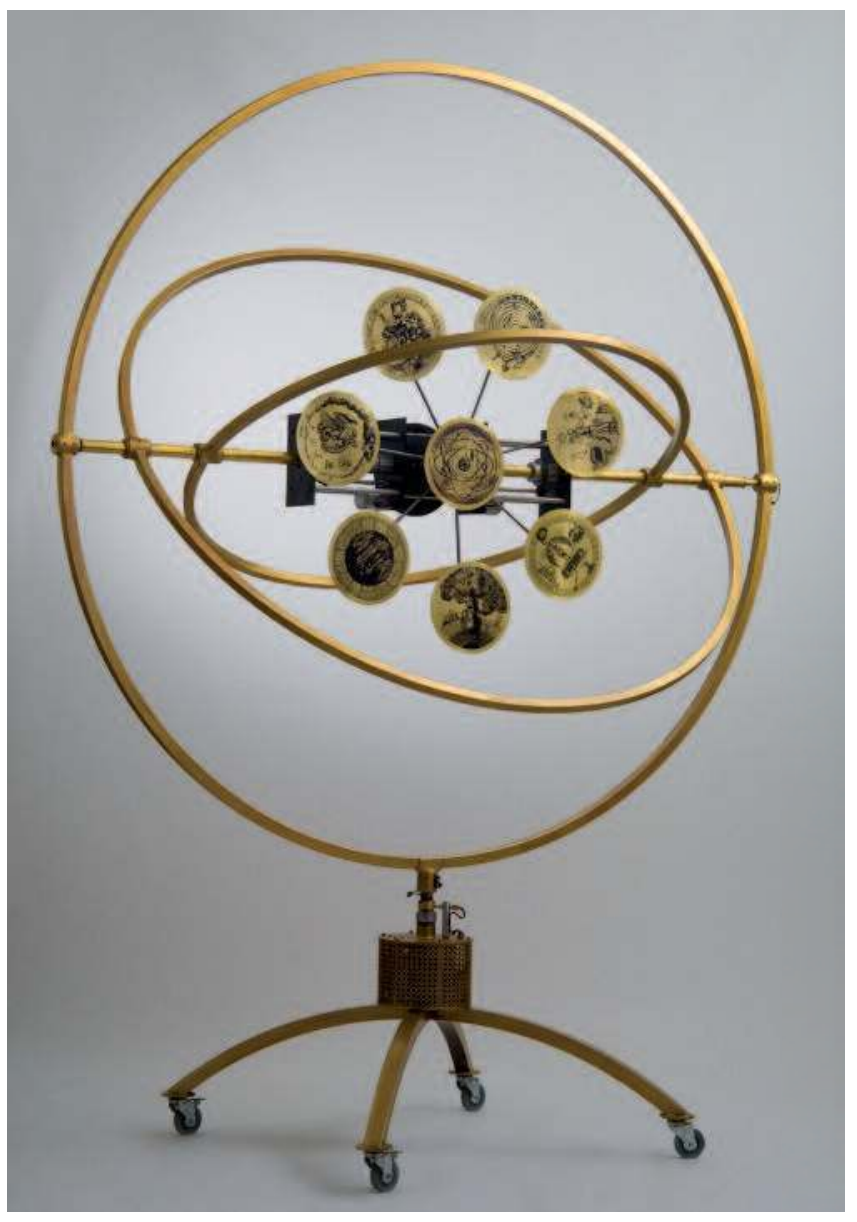

Horloge cosmique (dispositif mécanique et électronique en laiton et acier) par Piet's $\mathrm{O}$ et Peter Keene : les rotations, celle horizontale du grand anneau, celles verticales et opposées des deux anneaux intermédiaires, sont temporisées et inscrivent dans l'espace une géométrie variable de la pièce encadrant huit gravures centrales. () Isabel Tabelion et Roland Ménégon 
l'exposition se compose d'une ouvre et de la borne didactique destinée à l'accompagner et c'est l'ensemble de ces éléments qui est offert au public. Le rôle du commissaire de l'exposition (Jacques Lochard) a été de définir les thèmes du parcours didactique et de les structurer par rapport à l'histoire du siècle dernier, en particulier celle de l'évolution des principes de la radioprotection, en s'appuyant sur l'expertise du CEPN. Par ailleurs il a également assuré la médiation entre les artistes et les experts afin que s'instaure progressivement un véritable dialogue. Il s'agissait de concilier la volonté des artistes de tendre vers un certain « dépouillement », avec celle des experts de proposer une vision toujours plus détaillée. Le souci partagé par tous a été celui de la précision et de l'exactitude par rapport à la science. Cela n'a pas empêché les artistes de produire un travail de création particulièrement original.

\section{Art, alphabétisation et vulgarisation scientifique}

Jean-Yves BERNAUD : C'est la mission des centres de culture scientifique, technique et industrielle que d'être un creuset où viennent se mêler les visions et les apports des différents protagonistes intervenant lors de la conception et de la création d'un outil de vulgarisation. Les CCSTI ont vocation à faire de la vulgarisation scientifique, ainsi que de l'alphabétisation scientifique. Il en existe au moins un par région. La culture du grand public étant extrêmement limitée dans certains domaines scientifiques plus ou moins pointus, il est souvent nécessaire de procéder à une alphabétisation, avant d'envisager la vulgarisation. Il s'agit pour le grand public de comprendre et d'intégrer le langage scientifique et technique - c'est-à-dire de prendre la mesure des chiffres et des lettres destinés à porter le sens des travaux scientifiques. L'alphabétisation n'est certes pas particulièrement valorisante mais s'avère essentielle. Les spécialistes qui pensent pouvoir s'en affranchir s'enferment en réalité dans une culture dont eux seuls peuvent comprendre les tenants et les aboutissants.

Les centres de culture scientifique, technique et industrielle font régulièrement appel à des universitaires et à des spécialistes, en vue de partager, de diffuser et d'éclairer cette composante de notre culture contemporaine que présentent les domaines scientifiques et techniques. En région parisienne, le Palais de la Découverte et la Cité des Sciences et de l'Industrie constituent de formidables outils de médiation. En régions, les structures sont généralement plus modestes. Financées par l'État et les collectivités locales, elles n'existent qu'à travers la volonté politique des acteurs locaux. Mais que ce soit en province ou à Paris, trop peu font appel à des artistes pour intervenir dans la création d'outils de vulgarisation.

Dans notre cas, c'est parce que nous utilisons très souvent cette pratique, mais aussi parce que le domaine couvert par l'exposition Vous avez dit Radioprotection? Histoire de rayons $X$, de radioactivité et de radioprotection recouvrait un ensemble de thématiques, aussi complexes que sensibles, que nous avons considéré que l'approche artistique pouvait devenir un outil précieux de vulgarisation et d'appropriation. Le volet artistique devait toutefois être complété par un volet didactique. D'où l'idée d'une association entre des œuvres et des bornes didactiques. Par ailleurs l'équipe de production a préparé un catalogue de l'exposition qui présente les œuvres mais aussi une série de textes sur les diverses thématiques traitées par les vidéos.

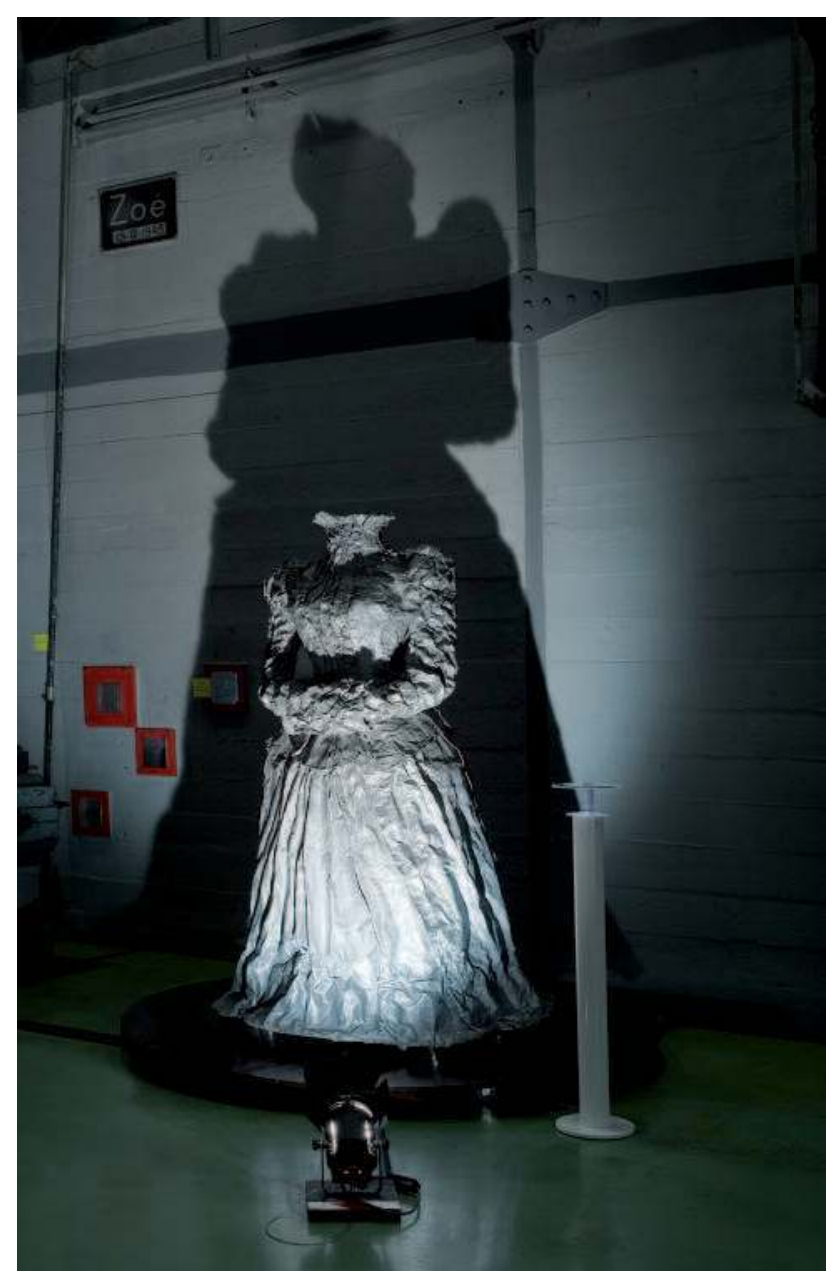

Dame de plomb (feuilles de plomb sur structure en bois et metal) par Piet'sO ๑ Isabel Tabelion et Roland Ménégon 
Jacques LOCHARD : Force est de constater que les supports vidéos fonctionnent bien dans le cadre de l'exposition. Cela étant, ils sont tout aussi efficaces lorsqu'ils sont projetés en dehors. J'ai eu l'occasion de les utiliser à des fins d'enseignement, avec des étudiants en troisième année d'école d'ingénieur. Ces derniers ont été captivés et ont réagi de manière tout à fait constructive. Du reste, ces films ont été conçus pour convenir également au grand public ainsi qu'à un public de scolaires.

En complément du catalogue, les visiteurs ont en effet la possibilité d'acquérir un DVD, contenant l'ensemble des supports, ainsi qu'un certain nombre de bonus. Ces derniers rassemblent toutes les informations plus ou moins vulgarisées n'ayant pas été reprises dans le produit final.

En outre, les supports didactiques ainsi réalisés constituent aujourd'hui la partie de l'exposition pouvant être diffusée le plus facilement en direction de tous les publics.

JYB : L'alphabétisation scientifique peut en effet débuter dès la maternelle. Cela fait partie de nos missions. Cela étant, le bon fonctionnement de l'exposition repose sur la présence obligatoire d'un animateur-médiateur. Ce dernier doit être doté d'une connaissance scientifique sûre et affirmer une réelle compétence théâtrale. Le point fort de ce système est de mettre en adéquation les outils de vulgarisation, avec les interrogations du public. Il s'agit, de surcroît, de faire émerger certaines questions que le public ne se pose pas nécessairement d'emblée. Notre ambition est de permettre au public de trouver des réponses grâce aux objets de l'exposition rendus « vivants » par le travail de l'animateur-médiateur. En cela, il porte une lourde responsabilité dans la réussite de l'action de développement culturel engagée.

Par ailleurs, afin d'élargir notre champ d'action dans le prolongement de l'exposition, nous travaillons à la mise en place de nouveaux outils : d'une part, le colporteur des sciences de la radioprotection à destination des élèves du primaire et des collèges, et, d'autre part, des ateliers de la radioprotection destinés au public lycéen. Les différents ateliers, constitués à l’intérieur des établissements scolaires, se réuniront une fois par an, en présence d'un certain nombre d'experts. L'exposition s'inscrit donc dans un dispositif complet de vulgarisation scientifique.

\section{De l'intime à l'Histoire}

Michel BRIÈRE : Les caractéristiques des rayons X et de la radioactivité rendent complexes les efforts de formation et de vulgarisation. De fait, la maîtrise des rayons $\mathrm{X}$ et de la radioactivité repose principalement sur des approches conceptuelles. Le niveau de formation des techniciens a dû être renforcé pour compenser l'absence de sensations associées aux phénomènes. Dans ce contexte, une approche artistique, pour partie conceptuelle, semble être de nature à accompagner l'effort de pédagogie en direction du grand public. Il s'agit ainsi de réduire l'écart entre la culture des ingénieurs et la culture du grand public. Nous accusons un certain retard dans ce domaine et il est regrettable que de telles initiatives n'aient pas été prises plus tôt.

JL : Il n'est jamais trop tard pour engager ce type de démarche. Du reste, votre remarque nous ramène au point de départ de l'exposition. Pour le grand public, l'art peut être un outil de médiation. Cependant, l'appropriation des concepts ne peut se faire qu'à partir des acquis dont chacun dispose. Chacun de nous développe une relation intime avec l'objet qu'est la

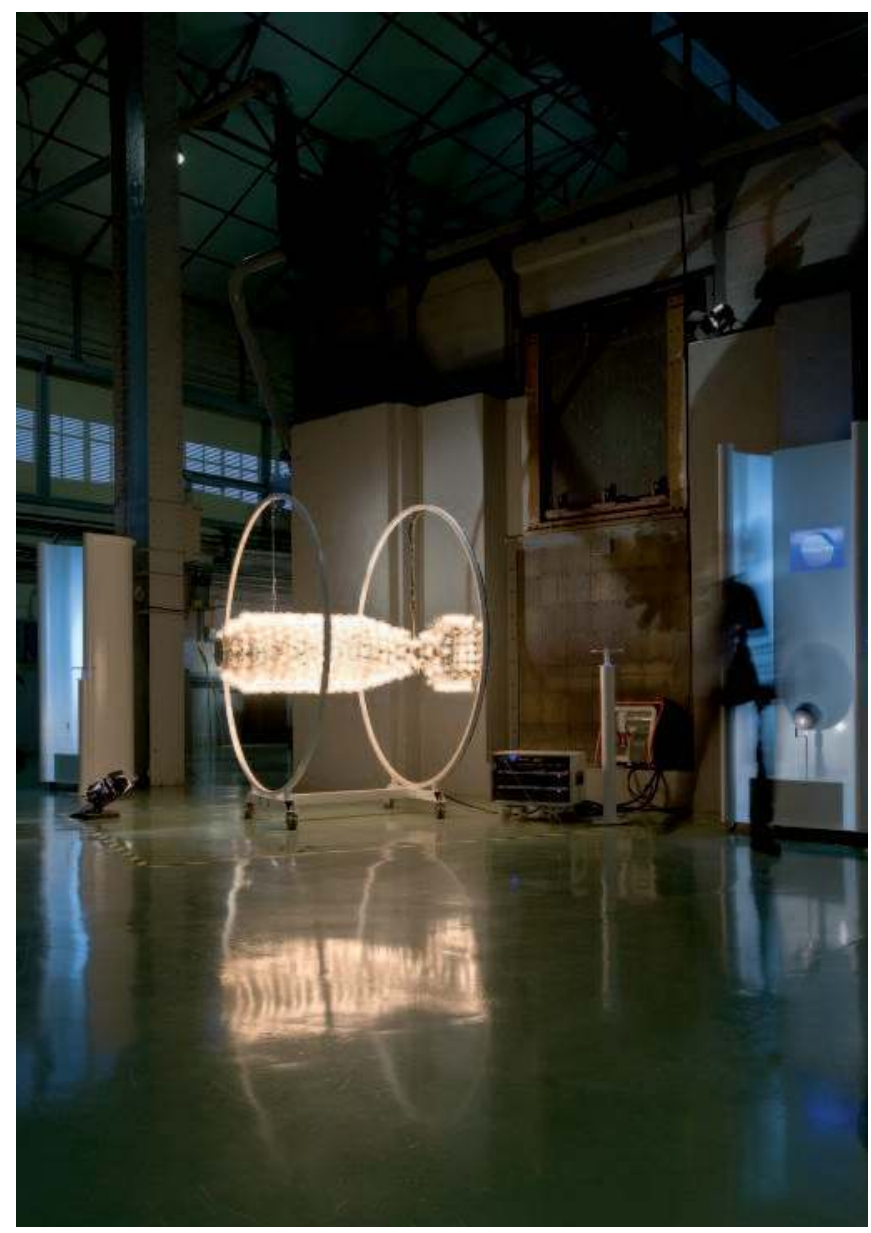

Little boy (dispositif électronique de contrôle et de séquençage formé d'une structure métallique et de 500 ampoules) par Peter Keene () Isabel Tabelion et Roland Ménégon 
radioactivité, en dépit du caractère insaisissable de cette dernière. Nous sommes partis de l'idée d'un chemin depuis l'intime jusqu'à la connaissance, à travers la médiation de l'art.

Les citoyens vivent dans l'intime leur relation avec la radioactivité. Cette dernière peut prendre un sens à travers par exemple des examens ou des actes médicaux qu'ils ont subis, le fait de vivre à proximité d'une installation nucléaire ou encore d'avoir participé au mouvement de lutte pour la paix. Chacun peut trouver en lui un fil qui le relie à la thématique en fonction de son vécu et son parcours personnel. Il s'agissait de toucher les visiteurs par ce biais. Tous ont une relation à l'objet, bien que la maîtrise de celui-ci demeure confisquée par les experts.

Lorsque les premiers contacts ont été établis en vue de créer l'exposition, il m’a été demandé de produire un texte court devant servir de base de réflexion à l'équipe qui serait en charge de produire l'exposition. J'y ai exposé cette idée de recourir à l'art pour convoquer l'intime et établir ainsi des liens avec les objets techniques.

Au départ, j’avais identifié un certain nombre d'œuvres qui semblaient renvoyer à l'objet de l'exposition. Un autoportrait d'Édouard Munch, tout d'abord, dont le bras squelettique semblait être inspiré des premières images de photographies aux rayons $\mathrm{X}$. Les formes expressives d'Henry Moore, exposées à Chicago devant la première pile atomique. Et enfin, la peinture déstructurée de Jackson Pollock qui, selon moi, symbolise la peinture de l'après Hiroshima.

Il m'avait semblé possible, à l'origine, de donner à voir ces œuvres, réunies autour d'une thématique commune. Cependant, il m'est apparu que la relation intime que chacun nourrit avec la radioactivité devait résonner avec l'Histoire. L'histoire des rayons $\mathrm{X}$ et de la radioactivité se déployait sur un siècle, ce qui devait pouvoir être structurant. Au-delà de l'écho des premières découvertes, il s'agissait de faire résonner en chacun le chemin parcouru dans le domaine médical, la rupture fondatrice de la fission... Il s'agissait également de renvoyer à la perception des deux catastrophes majeures liées à la radioactivité que sont Hiroshima et Tchernobyl. Le dialogue avec les artistes s'est construit autour de ces objets et de cette histoire.

JYB : L'exposition, en tant qu'objet, raconte une histoire. Du reste, nous ne pouvions pas décider d'emblée quels seraient les artistes associés à sa réalisation. Nous avons été amenés à faire des choix, sous le regard d'un comité scientifique. Il s'est agi de composer avec les sensibilités de chacun. Nous avons bien évidemment fait appel à nos réseaux pour solliciter un certain nombre d'artistes. Trois groupes d'artistes ont ainsi été constitués, auxquels le texte du commissaire d'exposition a été présenté. Chaque groupe a ensuite proposé un pré-projet.

Il fallait que les projets affichent une certaine proximité avec le message que nous souhaitions faire passer, tout en renvoyant à l'intime. Il convenait également que les artistes acceptent de s'associer à une démarche et à des mots porteurs de sens, liés à l'histoire des rayons $\mathrm{X}$, de la radioactivité et de la radioprotection. Le fait de s'associer à la démarche devait relever d'un engagement de leur part. Au-delà des choix du commissaire de l'exposition et de l'équipe, ils devaient souscrire à une logique de concertation, destinée à produire du sens.

L'équipe de production s'est, quant à elle, chargée de trouver des financements, de rassembler la documentation scientifique, de traduire l'information scientifique... Cet effort collectif nécessitait, de la

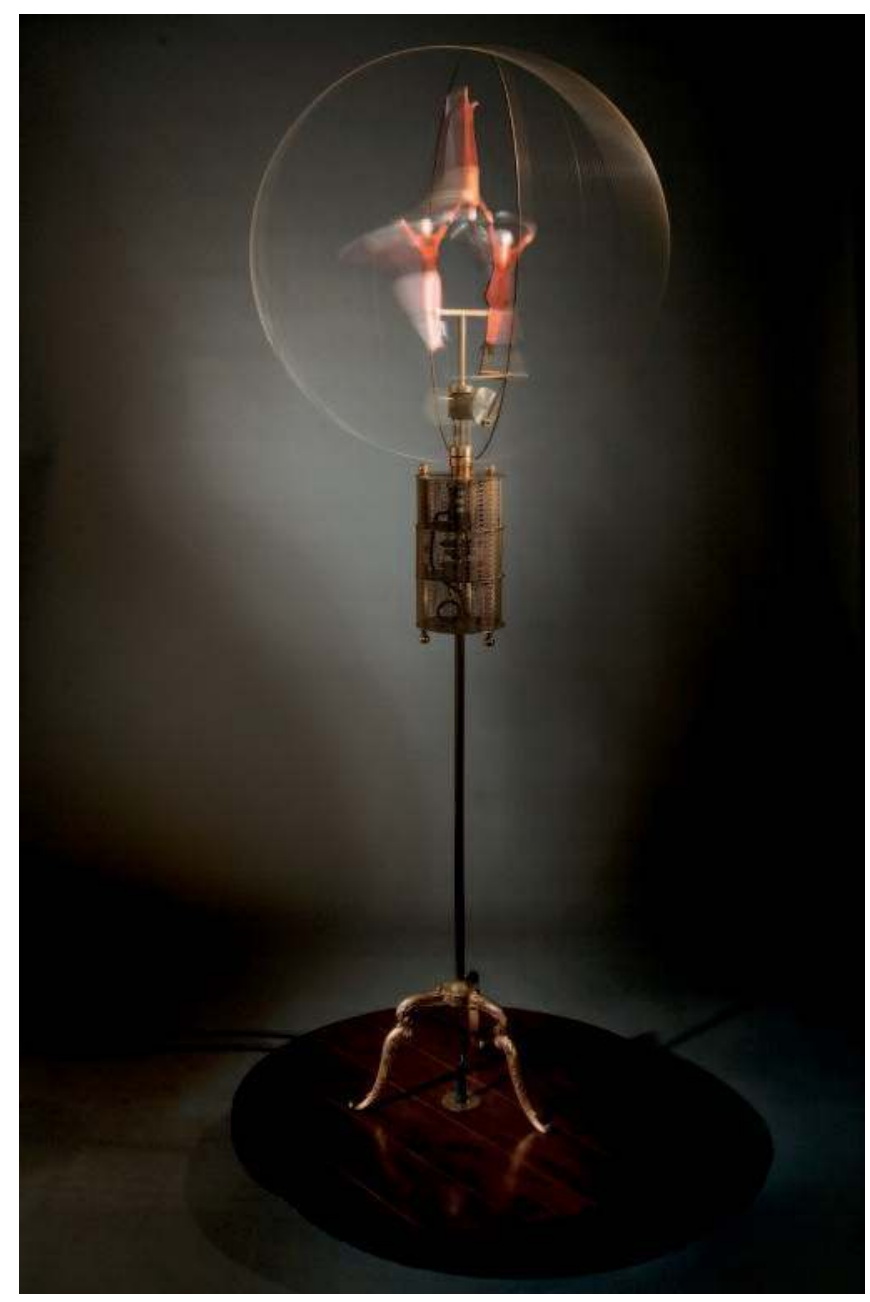

Fantastic Family Becquerel (dispositif mécanique et électronique en laiton et acier) par Peter Keene (c) Isabel Tabelion et Roland Ménégon 
part des artistes, une parfaite adhésion à l'équipe, mais aussi, une large ouverture d'esprit leur permettant d'intégrer nos remarques dans leur démarche de création artistique afin que leurs œuvres, donc leur sensibilité, leur intime, soient acceptés par tous.

En tout état de cause, la traduction de l'information scientifique en objets artistiques constituait véritablement un pari. De nombreux allers-retours ont été nécessaires avant de parvenir à un consensus, et si nous avons réussi, c'est parce que les artistes sont demeurés à l'écoute des remarques de chacun.

\section{L'artiste instrumentalisé ?}

MB : Les artistes n'ont-ils pas été gênés par le fait que leurs œuvres soient instrumentalisées ? De fait, il ne s'agit pas d'une démarche artistique conventionnelle. Nous touchons ici à la motivation profonde des artistes.

JL : Un des premiers croquis proposés par Peter Keene représentait l'œuvre aujourd'hui baptisée Little Boy. J'ai été immédiatement séduit par l'idée ayant toujours eu en tête la nécessité de ne pas occulter les rapports entre le nucléaire militaire et le nucléaire civil. En termes d'organisation et de communication, ces deux dimensions sont clairement distinguées par les institutions qui les gèrent. Il s'agit, de fait, de deux mondes à part. Cependant, le drame d'Hiroshima apparaît dans tous les esprits comme une sorte de "péché originel » du nucléaire civil, sur lequel repose un grand nombre d'amalgames. Le fait que Peter Keene propose de matérialiser ce lien entre la «bombe » et le nucléaire civil (représenté ici par l'électricité et le médical) permettait d'éclairer cette relation complexe qu'entretient l'individu avec l'objet. En s'appuyant sur une réelle connaissance des phénomènes scientifiques, Peter Keene est parvenu à incarner le sentiment de la plupart des citoyens dans son œuvre. Ce projet n'a pratiquement fait l'objet d'aucune discussion au sein de l'équipe. Il était entendu que le fait de poser ouvertement la question du rapprochement entre le civil et le militaire ne pouvait que susciter le débat et enclencher la réflexion des visiteurs. Aujourd'hui, cette pièce est une de celles qui les fascinent le plus, notamment les plus jeunes.

S'agissant des acrobates de la science qui illustrent l'épopée scientifique de la famille Becquerel, un consensus s'est également dégagé sans difficulté. En revanche, le traitement par les artistes de la catastrophe de Tchernobyl a donné lieu à des discussions bien plus conflictuelles. La première esquisse

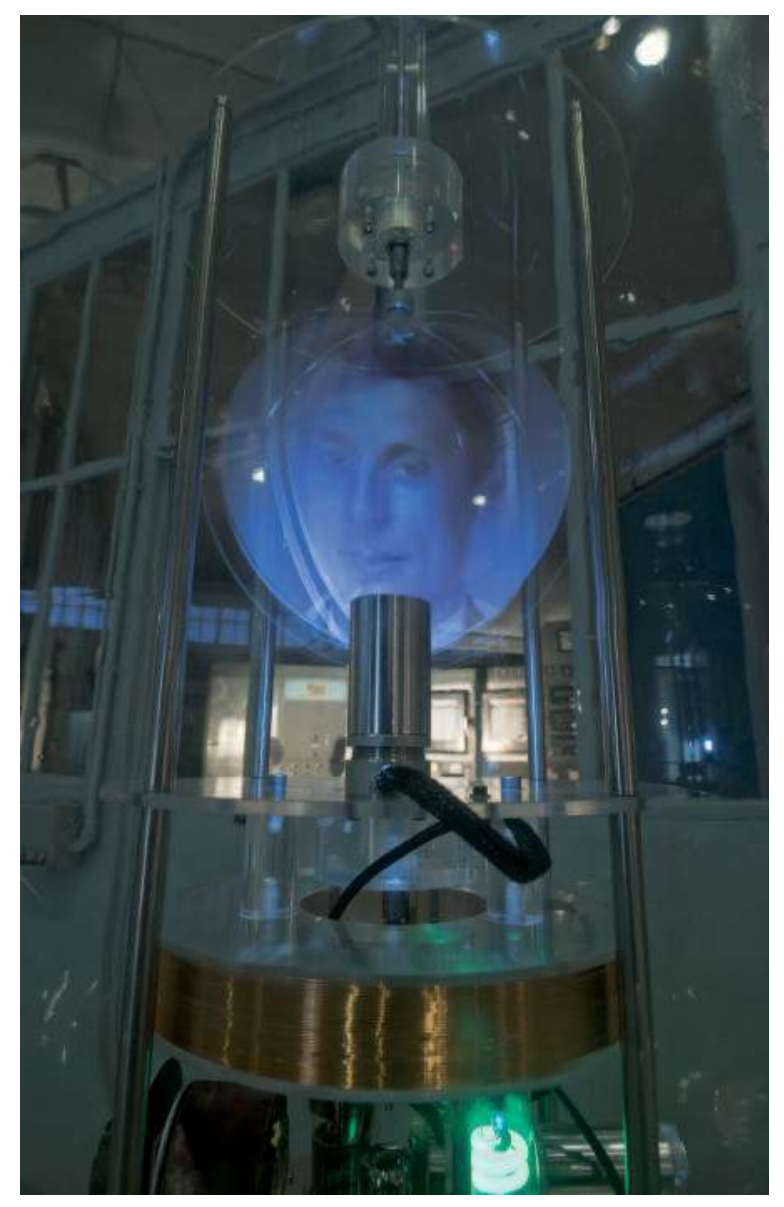

L'œuvre au rouge (réacteur en verre soufflé et acier, stroboscope) par Peter Keene () Isabel Tabelion et Roland Ménégon

représentait un théâtre de Guignol, avec en toile de fond un nuage radioactif. Nous ne pouvions accepter cette représentation quelque peu caricaturale car elle était trop réductrice du sens de la catastrophe. Il nous a fallu près de deux ans pour aboutir à un consensus. Le conflit était réel entre la volonté des artistes de revendiquer une certaine liberté de ton et la nécessité pour l'équipe de transmettre un message allant au-delà de la vision "simpliste » dans laquelle nous a enfermés la gestion de la catastrophe par les experts et les pouvoirs publics de l'époque.

Au terme d'un long processus, nous sommes finalement parvenus à forger un consensus. Nous ne pouvions envisager de consacrer une exposition à la radioactivité sans aborder la question de la catastrophe de Tchernobyl. Cependant, il nous fallait déjouer les stéréotypes véhiculés sur le sujet. Les artistes ont alors proposé un projet alternatif, baptisé Autant en emporte le vent. La confrontation nous a donc permis de dépasser les divergences et de faire évoluer ensemble le projet. Ce nouveau concept a tout de suite fait 
l'unanimité. Bien que ne disposant d'aucun recul, chacun y percevait du sens. L'œuvre permettait de traduire une réalité scientifique, tout en portant la réflexion au-delà de ses dimensions habituelles, sociétales et médiatiques. En outre, Piet'sO a su y adjoindre une bande son tout à fait extraordinaire et JeanYves Pipaud une vidéo éclairant parfaitement le sens de l'œuvre. La production de Autant en emporte le vent illustre le biais par lequel l'art peut jouer un rôle de médiation, à condition de trouver le bon « calage». La confrontation entre art et science nous a permis de dépasser les divergences.

JYB : Du reste, ce processus demeure cohérent avec celui de la pratique artistique. Rares sont les objets artistiques à être réalisés d'un seul coup. Chaque artiste travaille et retravaille son œuvre, avant de parvenir à un résultat pleinement satisfaisant de son point de vue. Ce mode de fonctionnement est davantage contraignant lorsqu'il s'applique à une équipe au sein de laquelle les vues et les objectifs peuvent être différents. La production de l'artiste cristallise les desiderata des uns et des autres. Celui-ci doit donc faire preuve de souplesse pour intégrer à sa propre réflexion esthétique les ambitions de chacun.

En tout état de cause, les œuvres devaient être pleinement abouties. De manière générale, une œuvre dont l'artiste n'est pas satisfait ne peut fonctionner, ni d'un point de vue artistique, ni dans une logique de médiation. La difficulté était dès lors de clore le processus de réflexion, au moment le plus opportun.

La question du devenir des œuvres devenant partie intégrante d'un objet de culture scientifique, telle une exposition, s'est également posée. L'enjeu pour les artistes était de continuer à intervenir sur l'exposition au cours de son itinérance, en fonction des contraintes de lieux et d'espaces. Il s'agissait de permettre aux artistes d'être satisfaits du rendu de l'exposition, telle que scénographiée en différents endroits.

De fait, les pièces n'ont jamais été disposées aléatoirement. La mise en scène de l'exposition revêt une importance capitale, en vue de recréer des parcours de sens. L'éclairage, pour ce faire, requiert également la plus grande attention. Il s'agit ainsi de faire naître des interactions entre l'objet et son environnement. C'est ensuite aux animateurs-médiateurs que revient le rôle d'aider à ce que ces interactions émergent à l'occasion de la confrontation du public aux œuvres. Au-delà de la connaissance scientifique, l'exposition devient ainsi un fait culturel.

\section{De l'expertise à la culture}

JL : Le rôle des animateurs-médiateurs dans l'exposition nous permet de réfléchir aussi sur la question du dialogue entre les experts et le grand public. Estce que finalement leur mission est de transmettre le savoir des experts sous une forme simplifiée qui serait ainsi plus accessible et plus assimilable ? L'expérience des animateurs sollicités dans la Drôme qui n'avaient pas été impliqués dans la production de l'exposition et qui ne bénéficiaient d'aucun support destiné à leur permettre de préparer leurs interventions montre que l'expertise n'est pas l'élément essentiel. En s'appuyant sur les interrogations du public, ils ont pu en quelques jours construire les éléments du dialogue et assurer la médiation. De fait, le public donne à partager sa relation intime avec l'objet. En ce sens, il contribue lui aussi à donner du sens à l'exposition.

À ce point, les difficultés rencontrées pour orchestrer le dialogue entre les membres de l'équipe de production et les experts doivent être évoquées : les uns souhaitaient tendre vers l'épure ; les autres vers le niveau de détail le plus fin. Une réflexion d'Hannah Arendt, tirée de la préface de son ouvrage La Condition de l'homme moderne, nous a guidés durant toute la période de préparation. L'auteur y pointe la nécessité pour les scientifiques de demeurer dans le «langage commun ». De fait, il ne saurait être reproché aux scientifiques d'avoir découvert le mécanisme de «la bombe », ni d'avoir été naïfs quant à l'utilisation qui pourrait en être faite. En revanche, il peut être reproché aux scientifiques de n'être pas demeurés dans le langage commun. Cette volonté de demeurer dans le langage commun a structuré nos échanges et structuré, en définitive, l'exposition elle-même. Nous avons ainsi fait le choix d'épurer au maximum les supports didactiques, en ne laissant filtrer que certains termes incontournables (becquerel, sievert...). Les rayons $\mathrm{X}$, la radioactivité et la radioprotection font aujourd'hui partie de notre monde (tant sur le plan pratique que sur le plan culturel) et ne semblent pas devoir en sortir. Il devient donc nécessaire d'organiser un dialogue autour de leurs enjeux, qu'il s'agisse des conséquences du développement de l'imagerie médicale ou de la politique énergétique des États. Les citoyens construisent au quotidien leurs relations avec ces objets et leurs enjeux. C'est pourquoi, nous ne pouvons nous retrancher dans un schéma au sein duquel seule une poignée d'experts seraient habilités à alimenter et à conduire les débats. 


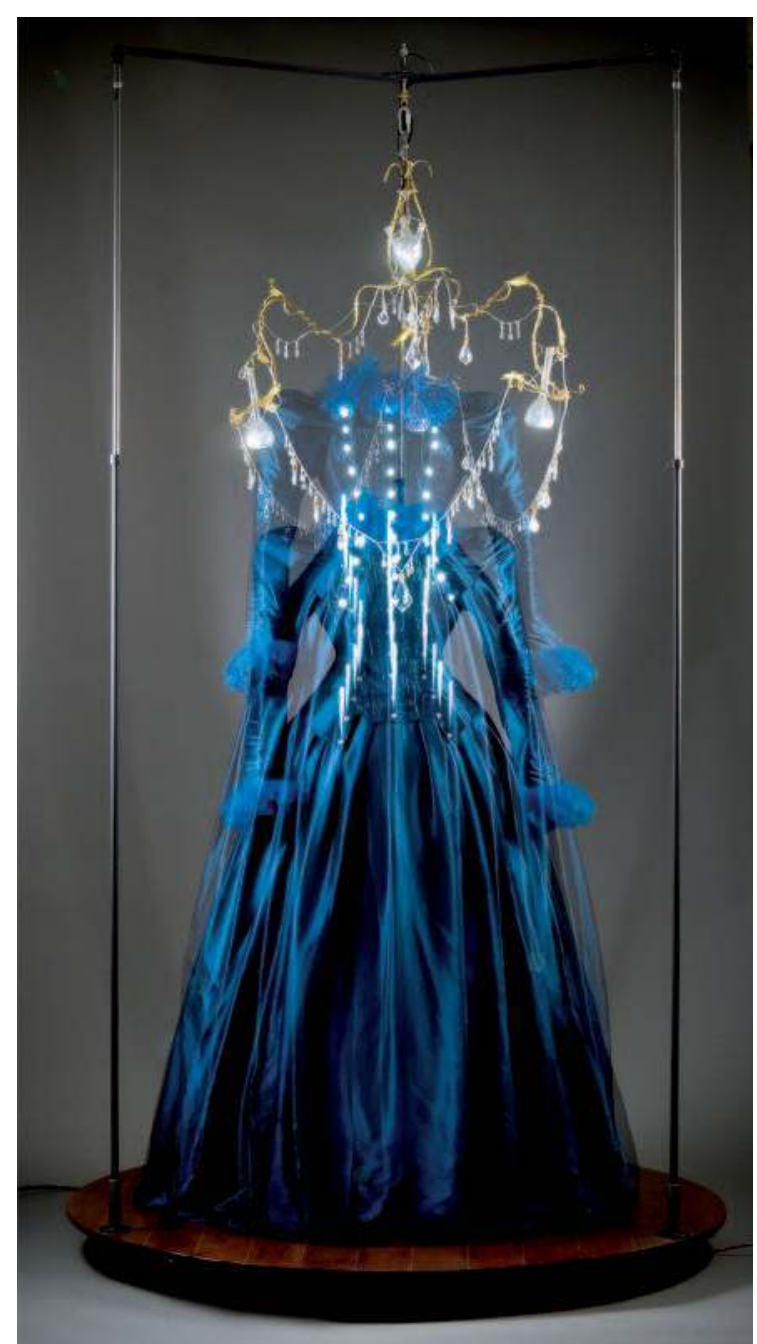

$X X-L$ (dispositif mécanique et électronique, en tissus, plexiglas, laiton, et diodes lumineuses) par $\mathrm{Piet}^{\prime} \mathrm{SO}$ : sous les applaudissements d'une bande sonore, la robe s'élève jusqu'au lustre et à son contact, les deux ensembles s'illuminent. () Isabel Tabelion et Roland Ménégon

MB : De fait, les questions qui touchent la radioactivité ne sauraient être abordées uniquement par le biais des sciences dures qui exigent entre autres des capacités à conceptualiser, mais doivent aussi passer par la médiation culturelle. Ces questions doivent donc trouver aussi un sens au travers de l'histoire, de la littérature, de la philosophie... L'exposition montre qu'une approche par la culture est possible à travers, par exemple les faits et les leçons de l'Histoire, y compris dans leurs dimensions les plus tragiques comme Hiroshima et Tchernobyl.

JYB : D'accord, mais il n'en demeure pas moins que le fait culturel est un acte complexe. La diffusion de la culture scientifique doit pouvoir s'appuyer sur la communication, l'enseignement et la vulgarisation.
Les centres de culture scientifique, technique et industrielle constituent un creuset dans lequel sont développés l'ensemble de ces axes, en vue de renforcer la culture des citoyens.

MB : L'information est transmise à travers la médiation de l'art. Cependant, le citoyen demeure seul juge de ce qu'il voit ou perçoit. L'objectif de la médiation doit être de développer l'accès à l'information, tout en laissant au citoyen la possibilité de forger ses propres représentations. L'adjonction d'un message est une entreprise tout à fait différente. Pour ma part, j'estime qu'il conviendrait de conserver comme objectif d'alimenter la culture scientifique des citoyens. L'objet de la médiation est précisément de provoquer l'échange. Dès lors, se pose la question du partage de l'information et des objets. Le rôle de la médiation va bien audelà de la communication.

JYB : La médiation est un acte global, qui prend son sens dans les codes de notre culture occidentale. Nous ne véhiculons ainsi que les codes et les présupposés de notre culture, et non pas ceux d'autres cultures.

JL : Un certain nombre de valeurs me semblent portées par l'Art et la Science, mais également par l'Éthique. Ces valeurs sont le produit de notre culture. C'est précisément là que se trouve le message qui, de fait, ne relève pas de la communication. La preuve en est que l'exposition, en tant qu'objet, ne peut guère être taxée de défendre telle ou telle idéologie. Il s'avère très difficile d'en remettre en cause la légitimité. Chacun peut y trouver des éléments de compréhension et/ou de justification. En réalité, l'exposition soulève des questions éthiques qui s'offrent au visiteur. Elle se veut propice à l'interprétation et non à l'instrumentalisation.

L'enjeu est dorénavant de développer une culture pratique de la radioprotection. S'agissant de prendre conscience collectivement de la dangerosité du radon, par exemple, le lien devrait être fait entre la diffusion d'une culture et les politiques de santé publique. Des politiques ont été mises en œuvre qui émanent d'experts, alors même que les phénomènes ne sont pas perceptibles par les populations. L'implication des populations ne pourra se faire qu'à travers la médiation. Aujourd'hui, la mesure même des dangers se trouve confisquée par les experts. Or la diffusion d'une culture pratique de la mesure pourrait faciliter grandement les opérations et réduire d'autant les coûts de protection associés pour la société. Elle permettrait surtout de développer la 
protection dans les domaines qui nécessitent la participation directe des personnes concernées par les expositions aux rayons $\mathrm{X}$ ou à la radioactivité.

MB : Le propre d'une culture est précisément de diminuer l'épaisseur des règlements. Lorsque l'implication des populations permet de démultiplier les actions, les prescriptions cessent d'être nécessaires. La culture de la radioprotection ne doit pas devenir une contrainte, mais bien au contraire susciter l'adhésion des populations.

JYB : La culture, à l'instar de la philosophie, est un espace de liberté qu'il nous appartient d'entretenir et de protéger.

\section{En guise de conclusion}

La science est en mouvement et l'écart se creuse doucement entre les recherches de pointe et la culture moyenne des citoyens. Dans ce contexte la vulgarisation scientifique est un exercice difficile. La schématisation et la simplification des principes et des découvertes, qui à première vue apparaissent nécessaires pour une compréhension par des non-spécialistes, non seulement introduisent une distorsion avec la réalité scientifique, mais, l'expérience le montre, contribuent le plus souvent à maintenir le grand public dans une posture de méfiance face aux objets de la science et la technique. Si l'objectif visé est de réduire cet écart, la course à la maîtrise des explications du monde est certainement une illusion.
Comment produire une iconographie et un narratif qui fassent sens et à partir desquels le visiteur pourra élaborer sa propre compréhension des enjeux de la science ? L'alphabétisation scientifique n'est pas soutenue par des « méthodes » comme l'apprentissage d'une langue. La mission du médiateur de culture scientifique est au contraire d'éclairer ces enjeux en offrant des angles d'observation qui intègrent l'observateur et permettent ainsi à une information complexe de se glisser dans son quotidien et d'être mise en perspective.

La culture scientifique accompagne une poétique de la relation au monde. Cette relation renforce le lien social en montrant la disponibilité du savoir si on l'approche librement. Cette poétique passe par un enchantement des sens. C'est là que l'expérience artistique est précieuse : fondée sur une insistance du regard, l'observation subjective des formes, l'histoire des représentations et la distance critique.

L'art, familier du geste gratuit, nous aide à poser sur la culture scientifique une lumière qui ne soit pas seulement fonctionnelle : la science se construit aussi sur des perceptions et des mystères. Face à la raison, une place est nécessaire pour la poésie, afin que la science ait quelque chance de croiser notre compréhension. 